PROCEEDINGS OF THE

AMERICAN MATHEMATICAL SOCIETY

Volume 139, Number 8, August 2011, Pages 2987-2993

S 0002-9939(2011)10876-1

Article electronically published on March 22, 2011

\title{
A CIRCLE IS NOT THE GENERALIZED INVERSE LIMIT OF A SUBSET OF $[0,1]^{2}$
}

\author{
ALEJANDRO ILLANES
}

(Communicated by Alexander N. Dranishnikov)

\begin{abstract}
In this paper we show that the simple closed curve cannot be obtained as the inverse limit of an upper semi-continuous multivalued function from $[0,1]$ into $[0,1]$.
\end{abstract}

\section{INTRODUCTION}

A continuum is a compact connected metric space with more than one point. We denote the Hilbert cube by $[0,1]^{\infty}$, and we consider $[0,1]^{\infty}$ with the metric

$$
d\left(\left(x_{1}, x_{2}, \ldots\right),\left(y_{1}, y_{2}, \ldots\right)\right)=\sum_{i=1}^{\infty} \frac{\left|x_{i}-y_{i}\right|}{2^{i}} .
$$

Given a closed subset $M$ of the square $[0,1]^{2}$, it is possible to define the generalized inverse limit of the set $M$ as

$$
\lim _{\leftarrow} M=\left\{\left(x_{1}, x_{2}, \ldots\right) \in[0,1]^{\infty}: \text { for each } i \in \mathbf{N},\left(x_{i+1}, x_{i}\right) \in M\right\} .
$$

When $M$ is the graph of a continuous function, $\lim _{\leftarrow} M$ is the usual inverse limit. In this case, it is well known that the continua that can be expressed as $\lim _{\leftarrow} M$ are exactly the chainable continua and, even for very simple maps, they can be extremely complicated; see for example [1].

Generalized inverse limits were introduced by W. S. Mahavier in [4, where he proved some basic properties and offered several interesting and illustrative examples. Generalized inverse limits are the natural notion of an inverse limit (or, in category theoretic terms, the limit) in the category whose objects are topological spaces, and where an arrow between $X$ and $Y$ is a closed subset of $X \times Y$, both of whose projections are onto. Even when these objects are very natural, they do not seem to be specifically studied from the categorical point of view.

An important problem in this area is:

Problem. What spaces can be obtained as $\lim _{\leftarrow} M$, for a closed subset $M$ of $[0,1]^{2}$ ?

Received by the editors October 8, 2009 and, in revised form, March 26, 2010.

2010 Mathematics Subject Classification. Primary 54C60; Secondary 54F15.

Key words and phrases. Inverse limits, Hilbert cube, simple closed curve, unit square, upper semi-continuous, set valued functions.

The author wishes to thank Gabriela Sanginés for her technical help during the preparation of this paper. 
In order to illustrate the variety of spaces that can be constructed with this technique, consider the following:

(a) If $M=[0,1] \times\left\{\frac{1}{2}\right\}$, then $\lim M$ is the singleton $\left\{\left(\frac{1}{2}, \frac{1}{2}, \ldots\right)\right\}$.

(b) If $M=\{(x, x): x \in[0,1]\}$, then $\lim M$ is an arc.

(c) If $M=([0,1] \times\{0,1\})$, then $\lim _{\leftarrow} \overleftarrow{M}$ is a Cantor set.

(d) If $M=[0,1]^{2}, \lim _{\leftarrow} M$ is the Hilbert cube.

(e) If $M=\{(x, x): x \in[0,1]\} \cup\{(x, 1-x): x \in[0,1]\}$, then $\lim _{\leftarrow} M$ is the cone over the Cantor set (Example 4 of [4]).

Of course, more interesting examples can be found in the references.

Given a closed subset $M$ of $[0,1]^{2}$, in general it is very difficult to visualize the space $\lim M$. This originated the comment of $\mathrm{W}$. S. Mahavier at the end of 4]: "All of our examples yield inverse limits that are either infinite dimensional or 1-dimensional continua. We suspect that this is true in general and that $\lim _{\leftarrow} M$ must either contain a Hilbert cube or be 1-dimensional." The answer to Mahavier's remark was given independently by A. Peláez in [5] and T. Ingram and W. S. Mahvier in [2]. They showed that for each positive integer $m$ it is possible to find a closed subset $M$ of $[0,1]^{2}$ such that $\lim M$ is $m$-dimensional.

In his talk in the Spring Topology and Dynamics Conference, 2009, Van C. Nall remarked that "It is not known if there is a one dimensional continuum that is not homeomorphic to an inverse limit with a single set valued function from $[0,1]$ to $[0,1]$. We will discuss some facts about a leading candidate, a simple closed curve." Later, in the Third Workshop in Continua and Its Hyperspaces, celebrated in the city of Toluca, in México (July, 2009), Van C. Nall gave a series of talks in which he showed several interesting examples and discussed his ideas about the problem of determining if a simple closed curve can be obtained as $\lim _{\leftarrow} M$, for some closed subset of $[0,1]^{2}$. This paper is the result of the discussions we had in this workshop, and the author wishes to thank all the participants. In particular, he wants to thank Professor Van C. Nall for transmitting his experience and expertise on this topic.

In this paper we prove that there is no closed subset $M$ of $[0,1]^{2}$ such that the projection of $M$ in both coordinates is onto and $\lim M$ is a simple closed curve.

A finite graph is a continuum $G$ which is the union of a finite number of arcs, each two of which intersect in a finite set. The following problem is open.

Problem. Does there exist a finite graph $G$, different from an arc, such that $G$ is homeomorphic to $\lim N$ for some closed subset $N$ of the square $[0,1]^{2}$ ?

\section{TwO AUXILIARY RESUlTS}

In this section we present two results that belong to the area of uniformization of functions. The ideas of the proofs come from the paper [6].

Let $\mathbf{N}$ be the set of positive integers. Given $n \in \mathbf{N}$, let $P_{n}=\{0,1, \ldots, n\}$.

Lemma 1. Let $f, g:[0,1] \rightarrow[0,1]$ be continuous functions such that $f(0)=0=$ $g(0)$ and $f(1)=1=g(1)$ and let $\delta>0$. Then there exist $n \in \mathbf{N}$ and functions $\alpha, \beta: P_{n} \rightarrow[0,1]$ such that $f \circ \alpha=g \circ \beta, \alpha(0)=0=\beta(0), \alpha(n)=1=\beta(n)$, and, for each $i \in\{0, \ldots, n-1\},|\alpha(i+1)-\alpha(i)|,|\beta(i+1)-\beta(i)|<\delta$. 
Proof. Let $A=\left\{(x, y) \in[0,1]^{2}: f(x)=g(y)\right\}$. The set $A$ is a compact subset of $[0,1]^{2}$ such that $(0,0),(1,1) \in A$. Let $L$ be the component of $A$ such that $(0,0) \in L$.

First, we prove that $(1,1) \in L$. Suppose to the contrary that $(1,1) \notin L$. Then there exist compact disjoint subsets $H$ and $K$ of $[0,1]^{2}$ such that $A=H \cup K$, $(0,0) \in H$ and $(1,1) \in K$. By [3, §57, III, Theorem 2, p. 438], there exists a separator $C$ between $(0,0)$ and $(1,1)$ which is a locally connected continuum disjoint from $A$. Thus there exist points $(x, y) \in C \cap((\{0\} \times[0,1]) \cup([0,1] \times\{1\}))$ and $(u, v) \in C \cap(([0,1] \times\{0\}) \cup(\{1\} \times[0,1]))$. Notice that $f(x) \leq g(y)$ and $f(u) \geq g(v)$. Since $C$ is connected, there exists a point $(t, s) \in C$ such that $f(t)=g(s)$. This implies that $(t, s) \in C \cap A$, a contradiction. Hence $(1,1) \in L$.

For each $(x, y) \in L$, let $U(x, y)=\left(x-\frac{\delta}{2}, x+\frac{\delta}{2}\right) \times\left(y-\frac{\delta}{2}, y+\frac{\delta}{2}\right)$. Consider the open cover $\mathcal{U}=\{U(x, y):(x, y) \in L\}$ of $L$. By the connectedness of $L$, there exist $n \in \mathbf{N}$ and points $\left(x_{1}, y_{1}\right), \ldots,\left(x_{n}, y_{n}\right) \in L$ such that $(0,0) \in U\left(x_{1}, y_{1}\right)$, $(1,1) \in U\left(x_{n}, y_{n}\right)$ and $U\left(x_{i}, y_{i}\right) \cap U\left(x_{i+1}, y_{i+1}\right) \cap L \neq \emptyset$ for each $i \in\{1, \ldots, n-1\}$. For each $i \in\{1, \ldots, n-1\}$, choose a point $\left(u_{i}, v_{i}\right) \in U\left(x_{i}, y_{i}\right) \cap U\left(x_{i+1}, y_{i+1}\right) \cap L$. Let $\alpha, \beta: P_{n} \rightarrow[0,1]$ be given by $\alpha(0)=0, \alpha(1)=u_{1}, \ldots, \alpha(n-1)=u_{n-1}$, $\alpha(n)=1, \beta(0)=0, \beta(1)=v_{1}, \ldots, \beta(n-1)=v_{n-1}$ and $\beta(n)=1$. Clearly, $\alpha$ and $\beta$ have the required properties.

Lemma 2. Let $f, g:[0,1] \rightarrow[0,1]$ be continuous functions such that $f(0)=0$ and $f(1)=1$ and let $\delta>0$. Then there exist $n \in \mathbf{N}$ and functions $\alpha, \beta: P_{n} \rightarrow[0,1]$ such that $f \circ \alpha=g \circ \beta, \beta(0)<\delta, 1-\beta(n)<\delta$ and, for each $i \in\{0, \ldots, n-1\}$, $|\alpha(i+1)-\alpha(i)|,|\beta(i+1)-\beta(i)|<\delta$.

Proof. Define $h:[0,1] \rightarrow[0,1]$ by

$$
h(t)= \begin{cases}3 t g(0), & \text { if } t \in\left[0, \frac{1}{3}\right], \\ g(3 t-1), & \text { if } t \in\left[\frac{1}{3}, \frac{2}{3}\right], \\ (3-3 t)(g(1))+3 t-2, & \text { if } t \in\left[\frac{2}{3}, 1\right] .\end{cases}
$$

Notice that $h$ is a well defined continuous function such that $h(0)=0$ and $h(1)=1$. Thus we can apply Lemma 1 and obtain $m \in \mathbf{N}$ and functions $\alpha_{1}, \beta_{1}$ : $P_{m} \rightarrow[0,1]$ such that $f \circ \alpha_{1}=h \circ \beta_{1}, \alpha_{1}(0)=0=\beta_{1}(0), \alpha_{1}(m)=1=\beta_{1}(m)$ and, for each $i \in\{0, \ldots, m-1\},\left|\alpha_{1}(i+1)-\alpha_{1}(i)\right|,\left|\beta_{1}(i+1)-\beta_{1}(i)\right|<\min \left\{\frac{1}{9}, \frac{\delta}{3}\right\}$.

Let $i_{0}=\max \left\{i \in\{0, \ldots, m\}: \beta_{1}(i) \in\left[0, \frac{1}{3}\right]\right\}$. Then $0<i_{0}<m$. Let $j_{0}=$ $\min \left\{j \in\left\{i_{0}, \ldots, m\right\}: \beta_{1}(j) \in\left[\frac{2}{3}, 1\right]\right\}$. Then $0<i_{0}+1<j_{0}-1<j_{0}<m$ and, for each $i \in\left\{i_{0}+1, \ldots, j_{0}-1\right\}, \beta_{1}(j) \in\left[\frac{1}{3}, \frac{2}{3}\right]$. Let $n=j_{0}-i_{0}-2$. Define $\alpha, \beta: P_{n} \rightarrow[0,1]$ by $\alpha(i)=\alpha_{1}\left(i+i_{0}+1\right)$ and $\beta(i)=3 \beta_{1}\left(i+i_{0}+1\right)-1$. Given $i \in\{0,1, \ldots, n\}, i+i_{0}+1 \in\left\{i_{0}+1, \ldots, j_{0}-1\right\}$, so $\beta_{1}\left(i+i_{0}+1\right) \in\left[\frac{1}{3}, \frac{2}{3}\right]$ and $f \circ \alpha(i)=f \circ \alpha_{1}\left(i+i_{0}+1\right)=h \circ \beta_{1}\left(i+i_{0}+1\right)=g\left(3 \beta_{1}\left(i+i_{0}+1\right)-1\right)=g \circ \beta(i)$. Thus $f \circ \alpha=g \circ \beta$.

Since $\beta(0)=3 \beta_{1}\left(i_{0}+1\right)-1$, by the definition of $i_{0}, \beta_{1}\left(i_{0}+1\right) \in\left[\frac{1}{3}, \frac{1}{3}+\frac{1}{9}\right)$, so $\beta(0) \in[0, \delta)$. Similarly, $\beta(n) \in(1-\delta, 1]$. It is easy to check that, for each $i \in\{0, \ldots, n-1\},|\alpha(i+1)-\alpha(i)|,|\beta(i+1)-\beta(i)|<\delta$.

\section{MAin RESUlt}

For each $i \in\{1,2\}$ let $\rho_{i}:[0,1]^{2} \rightarrow[0,1]$ be the $i$-th projection.

Throughout this section, $M$ will denote a closed subset of $[0,1]^{2}$ such that $\rho_{i}(M)=[0,1]$ for each $i \in\{1,2\}$. 
Given $x=\left(x_{1}, x_{2}, \ldots\right) \in[0,1]^{\infty}, A \subset[0,1]^{\infty}$ and $\varepsilon>0$, let $B(\varepsilon, x)$ be the $\varepsilon$ neighborhood around $x$ in $I^{\infty}$ and $N(\varepsilon, A)=\left\{x \in[0,1]^{\infty}\right.$ : there exists $a \in A$ such that $d(a, x)<\varepsilon\}$.

Theorem 1. There is no closed subset $M$ of $[0,1]^{2}$ such that $\lim _{\leftarrow} M$ is a simple closed curve.

Proof. Denote $S=\lim M$. Suppose that $S$ is a simple closed curve. For each $i \in \mathbf{N}$, let $\pi_{i}:[0,1]^{\infty} \stackrel{\leftarrow}{\rightarrow}[0,1]$ be the $i$-th projection. Since $\rho_{2}(M)=[0,1], \pi_{1}(S)=$ $[0,1]=\pi_{2}(S)$ and the projection $\pi_{1,2}: S \rightarrow M$ given by $\pi_{1,2}\left(\left(x_{1}, x_{2}, \ldots\right)\right)=\left(x_{1}, x_{2}\right)$ is onto, we obtain that $M$ is connected.

Let $\Delta$ be the diagonal of $[0,1]^{2}$; that is, $\Delta=\left\{(x, x) \in[0,1]^{2}: x \in[0,1]\right\}$. Given $x \in[0,1]$, we denote $\bar{x}=(x, x, \ldots) \in[0,1]^{\infty}$. Notice that if $(x, x) \in \Delta \cap M$, then $\bar{x}$ $\in S$. Given a map $\sigma:[0,1] \rightarrow[0,1]^{\infty}$ and $m \in \mathbf{N}$, let $\sigma_{m}:[0,1] \rightarrow[0,1]^{m}$ be the map given by $\sigma(t)=\left(\pi_{1}(\sigma(t)), \ldots, \pi_{m}(\sigma(t))\right)$. We consider the space $[0,1]^{m}$ with the metric $d_{m}\left(\left(x_{1}, \ldots, x_{m}\right),\left(y_{1}, \ldots, y_{m}\right)\right)=\sum_{i=1}^{m} \frac{\left|x_{i}-y_{i}\right|}{2^{i}}$.

Claim 1. If there exist $p, q \in S$ and $x \in[0,1]$ such that $\pi_{1}(p)=0, \pi_{1}(q)=1$ and $\bar{x}$ $\in S$, then $\bar{x} \in\{p, q\}$.

Suppose, contrary to Claim 1, that there exist $p, q \in S$ such that $\pi_{1}(p)=0$, $\pi_{1}(q)=1$ and $\bar{x} \in S-\{p, q\}$, where $x \in[0,1]$. Let $\sigma, \gamma:[0,1] \rightarrow S$ be one-toone continuous functions such that $\sigma(0)=p=\gamma(0), \sigma(1)=q=\gamma(1), \sigma\left(\frac{1}{2}\right)=\bar{x}$, $\operatorname{Im} \sigma \cap \operatorname{Im} \gamma=\{p, q\}$ and $\operatorname{Im} \sigma \cup \operatorname{Im} \gamma=S$.

Let $\varepsilon>0$ be such that $B(\varepsilon, \bar{x}) \cap \operatorname{Im} \gamma=\emptyset, N(\varepsilon, \operatorname{Im} \sigma) \cap N\left(\varepsilon, \gamma\left(\left[\frac{1}{3}, \frac{2}{3}\right]\right)\right)=$ $\emptyset, N\left(\varepsilon, \sigma\left(\left[0, \frac{1}{3}\right]\right) \cup \gamma\left(\left[0, \frac{1}{3}\right]\right)\right) \cap N\left(\varepsilon, \sigma\left(\left[\frac{1}{2}, 1\right]\right) \cup \gamma\left(\left[\frac{2}{3}, 1\right]\right)\right)=\emptyset$ and $N\left(\varepsilon, \sigma\left(\left[0, \frac{1}{2}\right]\right) \cup\right.$ $\left.\gamma\left(\left[0, \frac{1}{3}\right]\right)\right) \cap N\left(\varepsilon, \sigma\left(\left[\frac{2}{3}, 1\right]\right) \cup \gamma\left(\left[\frac{2}{3}, 1\right]\right)\right)=\emptyset$. Let $m \in \mathbf{N}$ be such that $\frac{1}{2^{m-1}}<\varepsilon$. Since $\operatorname{Im} \sigma$ is homeomorphic to $[0,1]$, there exists $\lambda>0$ such that $\lambda<\varepsilon$ and, if $d(\sigma(s), \sigma(t))<\lambda$, where $t, s \in[0,1]$, then diameter $(\sigma([s, t]))<\frac{\varepsilon}{2^{m-1}}$. Since the functions $\sigma_{m}:[0,1] \rightarrow[0,1]^{m}$ and $\gamma$ are continuous, there exists $\delta>0$ such that, if $t, s \in[0,1]$ and $|s-t|<\delta$, then $d_{m}\left(\sigma_{m}(s), \sigma_{m}(t)\right)<\frac{\lambda}{2}$ and $d(\gamma(s), \gamma(t))<\frac{\lambda}{2}$.

Since $\pi_{1}(\gamma(0))=\pi_{1}(p)=0$ and $\pi_{1}(\gamma(1))=\pi_{1}(q)=1$, we can apply Lemma 2 to the maps $\pi_{1} \circ \gamma$ and $\pi_{m} \circ \sigma$ and the number $\delta$, so there exist $n \in \mathbf{N}$ and functions $\alpha, \beta: P_{n} \rightarrow[0,1]$ such that $\pi_{1} \circ \gamma \circ \alpha=\pi_{m} \circ \sigma \circ \beta, \beta(0)<\delta, 1-\beta(n)<\delta$ and, for each $i \in\{0, \ldots, n-1\},|\alpha(i+1)-\alpha(i)|,|\beta(i+1)-\beta(i)|<\delta$.

Consider the function $\varphi: P_{n} \rightarrow S$ given by

$$
\varphi(i)=\left(\pi_{1}(\sigma(\beta(i))), \ldots, \pi_{m}(\sigma(\beta(i))), \pi_{2}(\gamma(\alpha(i))), \pi_{3}(\gamma(\alpha(i))), \ldots\right) .
$$

Given $i \in P_{n}$, we have that $\pi_{m}(\sigma(\beta(i)))=\pi_{1}(\gamma(\alpha(i)))$, then $\varphi(i) \in S$. Notice that $\sigma(\beta(i))$ and $\varphi(i)$ coincide in the first $m$ coordinates, so $d(\sigma(\beta(i)), \varphi(i)) \leq$ $\frac{1}{2^{m-1}}<\varepsilon$. By the choice of $\varepsilon, \varphi(i) \notin \gamma\left(\left[\frac{1}{3}, \frac{2}{3}\right]\right)$. Let $J=\gamma\left(\left[0, \frac{1}{3}\right]\right) \cup \operatorname{Im} \sigma \cup \gamma\left(\left[\frac{2}{3}, 1\right]\right)$. Then $J$ is a subarc of $S$ with end points $\gamma\left(\frac{1}{3}\right)$ and $\gamma\left(\frac{2}{3}\right)$ such that $\varphi\left(P_{n}\right) \subset J$. Consider the natural order $<$ on $J$ such that $\gamma\left(\frac{1}{3}\right)<\gamma\left(\frac{2}{3}\right)$.

Since $0 \leq \beta(0)<\delta$, we have that $d_{m}\left(\sigma_{m}(0), \sigma_{m}(\beta(0))\right)<\frac{\lambda}{2}<\frac{\varepsilon}{2}$. Since $\varphi(0)=\left(\pi_{1}(\sigma(\beta(0))), \ldots, \pi_{m}(\sigma(\beta(0))), \pi_{2}(\gamma(\alpha(0))), \pi_{3}(\gamma(\alpha(0))), \ldots\right)$, we have that $d(\sigma(0), \varphi(0))<\varepsilon$. This implies that $\varphi(0) \notin \sigma\left(\left[\frac{1}{2}, 1\right]\right) \cup \gamma\left(\left[\frac{2}{3}, 1\right]\right)$. That is, $\varphi(0)<$ $\bar{x}$. Similarly, $\bar{x}<\varphi(n)$. Thus there exists $i_{0}=\max \{i \in\{0,1, \ldots, n\}: \varphi(i) \leq \bar{x}\}$. Notice that $i_{0}<n$. 
Given $i \in\{0,1, \ldots, n-1\},|\alpha(i+1)-\alpha(i)|,|\beta(i+1)-\beta(i)|<\delta$. This implies that $d_{m}\left(\sigma_{m}(\beta(i)), \sigma_{m}(\beta(i+1))\right)<\frac{\lambda}{2}$ and $d(\gamma(\alpha(i)), \gamma(\alpha(i+1)))<\frac{\lambda}{2}$. Thus $d(\varphi(i), \varphi(i+1))<\lambda<\varepsilon$.

Since $\varphi\left(i_{0}\right) \in \gamma\left(\left[0, \frac{1}{3}\right]\right) \cup \sigma\left(\left[0, \frac{1}{2}\right]\right)$, by the choice of $\varepsilon, \varphi\left(i_{0}+1\right) \notin \sigma\left(\left[\frac{2}{3}, 1\right]\right) \cup \gamma\left(\left[\frac{2}{3}, 1\right]\right)$. Hence $\sigma\left(\frac{1}{2}\right)<\varphi\left(i_{0}+1\right)<\sigma\left(\frac{2}{3}\right)$. Similarly, $\sigma\left(\frac{1}{3}\right)<\varphi\left(i_{0}\right) \leq \sigma\left(\frac{1}{2}\right)$. In particular, $\varphi\left(i_{0}\right), \varphi\left(i_{0}+1\right) \in \operatorname{Im} \sigma$. Let $s, t \in[0,1]$ be such that $\varphi\left(i_{0}\right)=\sigma(s)$ and $\varphi\left(i_{0}+1\right)=$ $\sigma(t)$. Then $s \leq \frac{1}{2}<t$. By the choice of $\lambda$, diameter $(\sigma([s, t]))<\frac{\varepsilon}{2^{m-1}}$. Thus $d\left(\varphi\left(i_{0}\right), \bar{x}\right)<\frac{\varepsilon}{2^{m-1}}$. Hence,

$$
\begin{aligned}
\frac{1}{2^{m-1}} d\left(\gamma\left(\alpha\left(i_{0}\right)\right), \bar{x}\right) & =\frac{1}{2^{m-1}} \sum_{i=1}^{\infty} \frac{\left|\pi_{i}\left(\gamma\left(\alpha\left(i_{0}\right)\right)\right)-x\right|}{2^{i}} \\
& \leq \sum_{i=1}^{\infty} \frac{\left|\pi_{i}\left(\gamma\left(\alpha\left(i_{0}\right)\right)\right)-x\right|}{2^{m-1+i}}+\sum_{i=1}^{m-1} \frac{\left|\pi_{i}\left(\sigma\left(\beta\left(i_{0}\right)\right)\right)-x\right|}{2^{i}} \\
& =d\left(\varphi\left(i_{0}\right), \bar{x}\right) \\
& <\frac{\varepsilon}{2^{m-1}} .
\end{aligned}
$$

Therefore, $d\left(\gamma\left(\alpha\left(i_{0}\right), \bar{x}\right)\right)<\varepsilon$. This contradicts the choice of $\varepsilon$ and proves that Claim 1 holds.

Claim 2. $(0,0) \notin M$ or $(1,1) \notin M$.

Suppose, contrary to Claim 2 , that $(0,0),(1,1) \in M$. Then $\overline{0}, \overline{1} \in S$. Let $\sigma, \gamma:[0,1] \rightarrow S$ be one-to-one continuous functions such that $\sigma(0)=\overline{0}=\gamma(0)$, $\sigma(1)=\overline{1}=\gamma(1), \operatorname{Im} \sigma \cap \operatorname{Im} \gamma=\{\overline{0}, \overline{1}\}$ and $\operatorname{Im} \sigma \cup \operatorname{Im} \gamma=S$.

Let $\varepsilon>0$ be such that $N(\varepsilon, \operatorname{Im} \sigma) \cap N\left(\varepsilon, \gamma\left(\left[\frac{1}{3}, \frac{2}{3}\right]\right)\right)=\emptyset, N\left(\varepsilon, \sigma\left(\left[0, \frac{1}{3}\right]\right) \cup \gamma\left(\left[0, \frac{1}{3}\right]\right)\right)$ $\cap N\left(\varepsilon, \sigma\left(\left[\frac{1}{2}, 1\right]\right) \cup \gamma\left(\left[\frac{2}{3}, 1\right]\right)\right)=\emptyset$, and $N\left(\varepsilon, \sigma\left(\left[0, \frac{1}{2}\right]\right) \cup \gamma\left(\left[0, \frac{1}{3}\right]\right)\right) \cap N\left(\varepsilon, \sigma\left(\left[\frac{2}{3}, 1\right]\right) \cup\right.$ $\left.\gamma\left(\left[\frac{2}{3}, 1\right]\right)\right)=\emptyset$, and if $d(\gamma(s), \gamma(t))<\varepsilon$, then $|t-s|<\frac{1}{3}$. Let $m \in \mathbf{N}$ be such that $\frac{1}{2^{m-1}}<\frac{\varepsilon}{2}$. Let $L=\operatorname{Im} \sigma \cup \gamma\left(\left[0, \frac{1}{3}\right]\right) \cup \gamma\left(\left[\frac{2}{3}, 1\right]\right)$. Then $J$ is an arc. We consider $J$ with the natural order $<$ for which $\gamma\left(\frac{1}{3}\right)<\gamma\left(\frac{2}{3}\right)$. Given points $w, z \in J$, denote by $J(w, z)$ the subarc of $J$ joining them if $w \neq z$, and let $J(w, z)=\{w\}$ if $w=z$. Then there exists $\lambda>0$ such that $\lambda<\varepsilon$ and if $z, w \in J$ and $d(w, z)<\lambda$, then diameter $(J(w, z))<\frac{\varepsilon}{2^{m-1}}$. Let $\delta>0$ be such that $\delta<\frac{1}{3}$, and if $s, t \in[0,1]$ and $|s-t|<\delta$, then $d(\gamma(s), \gamma(t))<\frac{\lambda}{2^{m}}$ and $d(\sigma(s), \sigma(t))<\frac{\lambda}{2^{m}}$.

Since $\pi_{1}(\gamma(0))=\pi_{1}(\sigma(0))=\pi_{m}(\sigma(0))=0$ and $\pi_{1}(\gamma(1))=\pi_{1}(\sigma(1))=\pi_{m}(\sigma(1))$ $=1$, we can apply Lemma 1 to the pairs of maps $\left(\pi_{1} \circ \gamma, \pi_{m} \circ \sigma\right)$ and $\left(\pi_{1} \circ \sigma, \pi_{m} \circ \sigma\right)$ and to the number $\delta$, so there exist $n, k \in \mathbf{N}$ and functions $\alpha, \beta: P_{n} \rightarrow[0,1]$ and $\zeta, \eta: P_{k} \rightarrow[0,1]$ such that $\pi_{1} \circ \gamma \circ \alpha=\pi_{m} \circ \sigma \circ \beta, \pi_{1} \circ \sigma \circ \zeta=\pi_{m} \circ \sigma \circ \eta$, $\alpha(0)=\beta(0)=\zeta(0)=\eta(0)=0$, and $\alpha(n)=\beta(n)=\zeta(k)=\eta(k)=1$, for each $i \in\{0, \ldots, n-1\},|\alpha(i+1)-\alpha(i)|,|\beta(i+1)-\beta(i)|<\delta$ and, for each $j \in$ $\{0, \ldots, k-1\},|\zeta(j+1)-\zeta(j)|,|\eta(j+1)-\eta(j)|<\delta$.

Consider the functions $\varphi: P_{n} \rightarrow S$ and $\psi: P_{k} \rightarrow S$ given by

$$
\varphi(i)=\left(\pi_{1}(\sigma(\beta(i))), \ldots, \pi_{m}(\sigma(\beta(i))), \pi_{2}(\gamma(\alpha(i))), \pi_{3}(\gamma(\alpha(i))), \ldots\right)
$$

and

$$
\psi(j)=\left(\pi_{1}(\sigma(\eta(j))), \ldots, \pi_{m}(\sigma(\eta(j))), \pi_{2}(\sigma(\zeta(j))), \pi_{3}(\sigma(\zeta(j))), \ldots\right)
$$


Given $i \in P_{n}$, we have that $\pi_{m}(\sigma(\beta(i)))=\pi_{1}(\gamma(\alpha(i)))$; then $\varphi(i) \in S$. Notice that $\sigma(\beta(i))$ and $\varphi(i)$ coincide in the first $m$ coordinates, so $d(\sigma(\beta(i)), \varphi(i)) \leq$ $\frac{1}{2^{m-1}}<\varepsilon$. By the choice of $\varepsilon, \varphi(i) \notin \gamma\left(\left[\frac{1}{3}, \frac{2}{3}\right]\right)$. Thus $\varphi\left(P_{n}\right) \subset J$. Similarly, $\psi(j) \in S$, for each $j \in P_{k}$ and $\psi\left(P_{k}\right) \subset J$.

Given $i \in\{0,1, \ldots, n-1\},|\alpha(i+1)-\alpha(i)|,|\beta(i+1)-\beta(i)|<\delta$. This implies that $d(\sigma(\beta(i)), \sigma(\beta(i+1)))<\frac{\lambda}{2^{m}}$ and $d(\gamma(\alpha(i)), \gamma(\alpha(i+1)))<\frac{\lambda}{2^{m}}$. Thus $d(\varphi(i), \varphi(i+1))<\frac{\lambda}{2^{m-1}} \leq \lambda$. Hence diameter $(J(\varphi(i), \varphi(i+1)))<\frac{\varepsilon}{2^{m-1}}$. Similarly, for each $j \in\{0,1, \ldots, k-1\}$, diameter $(J(\psi(j), \psi(j+1)))<\frac{\varepsilon}{2^{m-1}}$.

Since $\varphi(0)=\overline{0}=\psi(0)$ and $\varphi(n)=\overline{1}=\psi(k)$, we can define $i_{0}=\max \left\{i \in P_{n}\right.$ : $\left.\varphi(i) \in \gamma\left(\left[0, \frac{1}{3}\right]\right)\right\}, i_{1}=\min \left\{i \in\left\{i_{0}, \ldots, n\right\}: \varphi(i) \in \gamma\left(\left[\frac{2}{3}, 1\right]\right)\right\}, j_{0}=\max \left\{j \in P_{k}:\right.$ $\left.\psi(j) \in \gamma\left(\left[0, \frac{1}{3}\right]\right)\right\}$ and $j_{1}=\min \left\{j \in\left\{j_{0}, \ldots, k\right\}: \psi(j) \in \gamma\left(\left[\frac{2}{3}, 1\right]\right)\right\}$. Notice that $i_{0}<$ $i_{1}$ and $j_{0}<j_{1}$. Notice also that, for each $i \in\left\{i_{0}+1, \ldots, i_{1}-1\right\}, \sigma(0)<\varphi(i)<\sigma(1)$. Since $d\left(\varphi\left(i_{0}\right), \varphi\left(i_{0}+1\right)\right)<\varepsilon$, by the choice of $\varepsilon, \varphi\left(i_{0}+1\right) \notin \sigma\left(\left[\frac{1}{2}, 1\right]\right) \cup \gamma\left(\left[\frac{2}{3}, 1\right]\right)$, so $\varphi\left(i_{0}+1\right) \in \sigma\left(\left[0, \frac{1}{2}\right)\right)$. Similarly, $\varphi\left(i_{1}-1\right) \in \sigma\left(\left(\frac{1}{2}, 1\right]\right)$. Thus $i_{0}+1<i_{1}-1$. Similarly, $j_{0}+1<j_{1}-1$. Notice that $\overline{0} \in J\left(\varphi\left(i_{0}\right), \varphi\left(i_{0}+1\right)\right)$. Thus $d\left(\overline{0}, \varphi\left(i_{0}+1\right)\right)<\frac{\varepsilon}{2^{m-1}}$. This implies that $d\left(\gamma(0), \gamma\left(\alpha\left(i_{0}+1\right)\right)\right)<\varepsilon$. Hence, $\alpha\left(i_{0}+1\right)<\frac{1}{3}$. Similarly, $\frac{2}{3}<\alpha\left(i_{1}-1\right)$.

Given $i \in\left\{i_{0}+1, \ldots, i_{1}-1\right\}, \psi\left(j_{0}\right) \leq \sigma(0)<\varphi(i)<\sigma(1) \leq \psi\left(j_{1}\right)$. Thus, there exists $j(i) \in\left\{j_{0}, \ldots, j_{1}\right\}$ such that $\psi(j(i)) \leq \varphi(i)<\psi(j(i)+1)$. Hence $d(\varphi(i), \psi(j(i)))<\frac{\varepsilon}{2^{m-1}}$. This implies that

$$
\begin{aligned}
\frac{1}{2^{m-1}} d(\gamma(\alpha(i)), \sigma(\zeta(j(i)))) & =\frac{1}{2^{m-1}} \sum_{l=1}^{\infty} \frac{\left|\pi_{l}(\gamma(\alpha(i)))-\pi_{l}(\sigma(\zeta(j(i))))\right|}{2^{l}} \\
& \leq d(\varphi(i), \psi(j(i))) \\
& <\frac{\varepsilon}{2^{m-1}} .
\end{aligned}
$$

Hence, $d(\gamma(\alpha(i)), \sigma(\zeta(j(i))))<\varepsilon$ and $\gamma(\alpha(i)) \in N(\varepsilon, \operatorname{Im} \sigma)$. By the choice of $\varepsilon$, $\alpha(i) \in\left[0, \frac{1}{3}\right] \cup\left[\frac{2}{3}, 1\right]$.

Therefore, $\alpha\left(i_{0}+1\right) \in\left[0, \frac{1}{3}\right], \alpha\left(i_{1}-1\right) \in\left[\frac{2}{3}, 1\right], \alpha(i) \in\left[0, \frac{1}{3}\right] \cup\left[\frac{2}{3}, 1\right]$ for each $i \in\left\{i_{0}+1, \ldots, i_{1}-1\right\}$ and $|\alpha(i+1)-\alpha(i)|<\delta<\frac{1}{3}$ for each $i \in\left\{i_{0}+1, \ldots, i_{1}-2\right\}$. Clearly, this is absurd. We have shown that Claim 2 holds.

We are ready to obtain a final contradiction. Since $\pi_{1}(S)=[0,1]=\pi_{2}(S)$, there exist points $p, q \in S$ such that $\pi_{1}(p)=0$ and $\pi_{1}(q)=1$. Since $M$ is connected and $\rho_{i}(M)=[0,1]$ for each $i \in\{1,2\}$, we have that $M \cap \Delta \neq \emptyset$. Let $x \in[0,1]$ be such that $(x, x) \in M \cap \Delta$. Then $\bar{x} \in S$. By Claim $1, \bar{x} \in\{p, q\}$. If $\bar{x}=p$, then $0=\pi_{1}(p)=\pi_{1}(\bar{x})$, so $x=0$ and $p=\overline{0}$. Similarly, if $\bar{x}=q$, then $\bar{x}=\overline{1}$ $=q$. Therefore, $\bar{x} \in\{\overline{0}, \overline{1}\}$. This shows that $M \cap \Delta \subset\{(0,0),(1,1)\}$. By Claim 2, $(0,0) \notin M$ or $(1,1) \notin M$. Thus $M \cap \Delta=\{(0,0)\}$ or $M \cap \Delta \subset\{(1,1)\}$. We may assume that $M \cap \Delta=\{(0,0)\}$; the other case is similar. Notice that $\overline{0} \in S$.

Let $T=\left\{r \in S: \pi_{1}(r) \geq \pi_{2}(r)\right\}$ and $R=\left\{r \in S: \pi_{1}(r) \leq \pi_{2}(r)\right\}$. Then $T$ and $R$ are closed subsets of $S$ such that $S=T \cup R$ and $\{\overline{0}\} \subset T \cap R$. If $r=\left(r_{1}, r_{2}, r_{3}, \ldots\right) \in T \cap R$, then $r_{1}=r_{2}$. Thus $\left(r_{2}, r_{1}\right) \in M \cap \Delta$. Hence $r_{1}=0=r_{2}$. If $r \neq \overline{0}$, we can take $m=\min \left\{i \in \mathbf{N}: r_{i} \neq 0\right\}$. Then $m \geq 3$; the point $p_{0}=\left(r_{m-1}, r_{m}, r_{m+1}, \ldots\right)$ belongs to $S$ and $\pi_{1}\left(p_{0}\right)=0$. Applying Claim 1 to $p_{0}, q$ and $\overline{0}$, we obtain a contradiction. Therefore, $r=\overline{0}$. We have shown that $T \cap R=\{\overline{0}\}$. 
Since $\rho_{i}(M)=[0,1]$ for each $i \in\{1,2\}$, we have that $T-\{\overline{0}\} \neq \emptyset$ and $R-\{\overline{0}\} \neq \emptyset$. Thus 0 is a cut point of $S$. This is impossible since $S$ is a simple closed curve. This finishes the proof of the theorem.

\section{REFERENCES}

[1] W.T. Ingram, Inverse limits on [0,1] using piecewise linear unimodal bonding maps, Proc. Amer. Math. Soc. 128 (2000) 287-297. MR1618702 (2000c:54025)

[2] W.T. Ingram; W.S. Mahavier, Inverse limits of upper semi-continuous set valued functions, Houston J. Math. 32 (2006), 119-130. MR.2202356 (2006i:54020)

[3] K. Kuratowski, Topology, vol. 2, Academic Press and PWN, New York, London and Warzawa, 1968. MR0259835 (41:4467)

[4] W.S. Mahavier, Inverse limits with subsets of $[0,1] \times[0,1]$, Topology Appl. 141 (2004), 225231. MR2058690(2005c:54012)

[5] A. Peláez, Generalized inverse limits, Houston J. Math. 32 (2006), 1107-1119. MR 2268473 (2007m:54009)

[6] R. Sikorski and K. Zarankiewicz, On uniformization of functions. I, Fund. Math. 41 (1955), 339-344. MR0072465 (17:288g)

Instituto de Matemáticas, Universidad Nacional Autónoma de México, Circuito Exterior, Ciudad Universitaria, México 04510, D.F.

E-mail address: illanes@matem. unam.mx 